\title{
La conceptualización del espacio: Desarrollo y sistemas de referencia
}

\section{The conceptualization of space: development and reference systems}

DOI: https://doi.org/10.32870/dse.v0i22.700

\author{
Lidia Lozada Nava* \\ Gabriela Calderón Guerrero**
}

\begin{abstract}
Resumen
La sobrevivencia humana depende en gran medida de comunicar a otros lo que se piensa sobre el espacio, de ahí su importancia como objeto de conocimiento y de reflexión escolar. El espacio, como lo señalan Levinson y Wilkins (2006), ha sido culturalmente organizado a través de distintos tipos de sistemas de referencia y deícticos. Para Piaget e Inhelder (1973), la capacidad de coordinar coherentemente la información en un todo congruente es una de las características más importantes del desarrollo del conocimiento. El propósito de este trabajo es analizar cómo se desarrolla la conceptualización del espacio en la infancia y la adolescencia, y si dicho desarrollo se ve afectado por la capacidad de coordinar coherentemente la información. A 60 niños y adolescentes mexicanos se les propuso la tarea de describir una escena visual para que otra persona, siguiendo sus instrucciones, acomodara una serie de objetos en una maqueta. Los resultados indican que los alumnos entrevistados muestran diferencias sistemáticas y significativas en la conceptualización y descripción del espacio, y estas se relacionan con la creciente capacidad de coordinar las pistas y la información. Asimismo, el trabajo ilustra la utilidad del análisis lingüístico como herramienta para llevar a cabo investigación educativa.
\end{abstract}

Palabras clave: Espacio - desarrollo - sistemas de referencia - deixis - coordinación de información.

\begin{abstract}
Human survival depends largely on communicating to others our conceptualization of space, hence its importance as an object of knowledge and school reflection. Space, as Levinson and Wilkins (2006) point out, has been organized culturally through different types of reference systems and deixis. According to Piaget and Inhelder (1973), the ability to coherently coordinate information in a congruent whole is one of the most important characteristics of the development of knowledge. This paper see-
\end{abstract}

* Maestra en Aprendizaje de la Lengua y las Matemáticas. Líneas de investigación: Adquisición de la lengua escrita, cuerpo, movimiento y cognición. Universidad Autónoma de Querétaro. México. lidiaylolita@gmail.com

* Doctora en Lingüística. Líneas de investigación: Desarrollo y construcción de conocimiento, adquisición de la lengua escrita, educación y trabajo, género y educación, acoso escolar. Universidad Autónoma de Querétaro. México. gabygro2000@yahoo.com 
ks to analyze how the conceptualization of space develops in childhood and adolescence, and how it is affected by the ability to coordinate information coherently. 60 Mexican children and adolescents were asked to describe a visual scene so another person, following their instructions, could arrange a series of objects in a model. The results indicate that the students interviewed show systematic and significant differences in the conceptualization and description of the space and these are related to the increasing ability to coordinate clues and information. Also, the work illustrates the usefulness of linguistic analysis as a tool to carry out educational research.

Keywords: Space - development - reference systems - deixis - cognitive coordination.

\section{Introducción}

El estudio y análisis de qué es el espacio y cómo se desarrolla dicha noción en la infancia y la adolescencia resulta altamente relevante, entre otras razones, porque la sobrevivencia humana depende en buena medida de su conceptualización y de su interpretación simbólica adecuada (Chatterjee, 2001).

La noción de espacio es un concepto altamente complejo que atañe prácticamente a todas las dimensiones del ser-desde la biofísica, la psicológica, la psicolingüística, hasta las socioculturales, las ideológicas y las económicas, entre otras- (Calderón, Lozada, 2020; Werlen, 2017); de esta forma, no es de extrañar que haya sido motivo de interés para diversas disciplinas y tradiciones del pensamiento a lo largo de la historia humana.

Para Moliner (2007: 1244), el espacio puede ser entendido como"la magnitud en que están contenidos todos los cuerpos que existen al mismo tiempo y en la que se miden esos y la separación entre ellos". Desde el punto de vista psicológico, el espacio es considerado como la proyección originada en el cuerpo que se extiende en todas direcciones hasta el infinito (Piaget, Inhelder, 1947).

En esta misma línea de pensamiento, Lurcat (2014) señala que la conceptualización del espacio es posible gracias al uso de referencias espaciales. Al respecto, Calderón y Lozada señalan:

La referencia espacial permite establecer la ubicación de objetos, lugares o personas en el espacio y tiene como fuente la actividad sensorial y motora de los sujetos (fuente directa), así como la información sociocultural de la comunidad (fuente indirecta) (Lurcat, 2014). Ambas fuentes se desarrollan progresivamente en muy estrecha relación... las fuentes son personales y las referencias pueden ser muy distintas entre individuos debido a la experiencia y conocimiento del espacio, así como del propio esquema corporal, de ahí la importancia de estudiar su desarrollo a nivel ontogenético e individual (2020: 80).

Por su parte, Milton Santos, geógrafo que con visión crítica pensó sobre el espacio como constructo que responde también a problemáticas e intereses sociales, culturales y humanas, lo consideró como una instancia ideológico-cultural, además de geográfica; es decir, Santos 
planteó una ontología del espacio en tanto visualizó y analizó de forma crítica las diversas nociones fundantes de su ser, entre las que se encuentra el tiempo (Panadero, 2000). Esta perspectiva crítica le permitió plantear que la construcción social que se hace del espacio genera desigualdades, que a su vez son reforzadas por lo que se piensa y se dice de este en un colectivo social. Santos consideraba que una sociedad solo es cuando está siendo, realizándose. La sociedad se teje sobre el espacio y su uso, el tiempo y su uso, que constituyen su base material. Para los humanos, el tiempo solo es asible empirizándolo, volviéndolo material, y únicamente a través de esta materialización del tiempo podemos asimilar el espacio a nuestros esquemas (Panadero, 2000).

En una línea de análisis similar, Werlen (2017) plantea que a las realidades socioculturales siempre les subyacen relaciones de tipo espacial. Si una sociedad no es capaz de detectar y reconocer este tipo de relaciones espaciales que tejen la ontología profunda del colectivo, se pueden generar o encubrir profundos conflictos políticos, raciales, ecológicos, entre otros (Calderón, Lozada, 2020).

No obstante, a pesar de la importancia que desde diversas ciencias se le reconoce al escudriñamiento de esta noción, en el campo educativo mexicano prácticamente no existen investigaciones que den cuenta de cómo se desarrolla este concepto en la infancia y la adolescencia a nivel biofísico (desplazamiento y ubicación de objetos y de uno mismo en el espacio físico y en el espacio geométrico) (Maravall, 2007), cognoscitivo y psicolingüístico. Tampoco existen trabajos que documenten el impacto de las metáforas espaciales (como "arriba es bien, abajo es mal") en el pensamiento infantil y adolescente, metáforas altamente empleadas en nuestro sistema de pensamiento y en nuestra ideología moderna, así como en el sistema de producción económica capitalista, y que son asumidas en el imaginario colectivo, reforzando esquemas binarios y antagónicos de relación social con los otros (por ejemplo, en la dualidad de "los de arriba" y "los de abajo"). Si bien este trabajo se centrará tan solo en la noción de espacio y su relación con el desarrollo cognoscitivo y los sistemas de referencias expresados en la lengua, la necesidad de estudios en torno al espacio, su importancia y su relación con las áreas ya mencionadas es ineludible.

En el caso concreto del presente trabajo, nuestras aportaciones pueden ayudar a entender la relación espacio, cognición y lenguaje en el desarrollo de niños y jóvenes mexicanos, lo que a su vez podría contribuir a la toma de decisiones pedagógicas y didácticas con base en información recabada en investigaciones realizadas en nuestro país.

En la siguiente sección se expondrán los conceptos elementales para entender a qué nos referimos cuando hablamos de sistemas de referencia y deixis como aspectos fundamentales de la conceptualización espacial. Posteriormente, se señalarán algunas características generales del desarrollo cognoscitivo, la coordinación de información y del desarrollo cognoscitivo espacial (Delval, 1995; Piaget, Inhelder, 2015). 


\section{Sistemas de referencia y deixis en la conceptualización del espacio}

Según Levinson (1996; 2003), Levinson y Wilkins (2006) y Pederson, Danziger, Wilkins, Levinson, Kita y Senft (1998), el espacio se conceptualiza gracias a las referencias que somos capaces de establecer. Esas referencias se organizan en sistemas (sistemas de referencias) que han sido creados socioculturalmente y cuyos conceptos se expresan en la lengua de manera fundamental a través de los deícticos, aunque no únicamente. Los sistemas de referencia espacial más importantes que existen son: $a)$ los sistemas sin coordenadas y b) los sistemas con coordenadas, que dividen el espacio en ejes o áreas imaginarias y de los que existen dos subtipos: sistemas con coordenadas cartesianas (eje $\mathrm{Y}, \mathrm{X}$ y $Z$ ) y sistemas con coordenadas de tipo geocéntrico (norte, sur, este, oeste, etc.). Cabe señalar que un gran porcentaje de las lenguas indoeuropeas utilizan sistemas de referencia de tipo cartesiano y solo en contextos especializados se tiende a usar las referencias geocéntricas (Janzen, Haun, Levinson, 2012; Levinson, Wilkins, 2006; Majid, Bowerman, Kita, Haun, Levinson, 2004).

El sistema de organización espacial sin coordenadas establece la referencia de forma altamente contextualizada, por lo que emplea deícticos pronominales del tipo aquí, allá, al lado de, locuciones adverbiales locativas, marcas de contigüidad y relaciones topológicas (en la mesa, en la silla), así como nombres de lugares (por ejemplo, Querétaro). Por su parte, el sistema de referencia con coordenadas de tipo cartesiano toma como referente los ejes imaginarios cartesianos, que no son otra cosa que abstracciones del espacio; los deícticos de los que se vale este tipo de sistema son: en el plano vertical (eje Y) arriba-abajo, en el plano horizontal (eje X) derecha-izquierda y la profundidad (eje Z) adelante-atrás.

Levinson y Wilkins (2006) señalan que representar el espacio exclusivamente a través de un sistema de organización espacial sin coordenadas, implica un menor reto cognitivo de lo que suponen los sistemas con coordenadas, ya que estos últimos requieren mayores capacidades de abstracción y descontextualización de la información, dado que lo que representan son fundamentalmente relaciones espaciales y no locaciones concretas.

A propósito del reto cognitivo, definir una localización en el eje vertical u horizontal en gran medida dependerá de la variación de esta cuando la persona se mueve (Franklin, Tversky, 1990). En ese sentido, definir las coordenadas en el plano vertical resulta más sencillo pues, aunque la persona se desplace en el ambiente, la ubicación vertical relativa al observador (arribaabajo) se mantiene bastante constante (Shepard, Hurwitz, 1984; Tommasi, Laeng, 2012). Por el contrario, las dimensiones horizontales cambian con el movimiento y, por tanto, son más difíciles de abstraer, comprender y representar. Dentro de estas, la dimensión delante-detrás es menos compleja pues se correlaciona con la dirección normal del movimiento, y con la asimetría existente entre el frente del propio cuerpo y la parte trasera del mismo (el frente es visible) (Levinson, 2003). Por su parte, la dimensión derecha-izquierda es más difícil puesto que no se 
correlaciona con la ruta normal del movimiento, sino que se basa en las mitades izquierda y derecha del cuerpo, las cuales son relativamente simétricas (Levinson, 1996).

Es necesario enfatizar que los sistemas de referencia necesitan el fenómeno de la deixis para organizar y conceptualizar el espacio. Los deícticos solamente adquieren significado pleno en el contexto en que se emiten, por ello son elementos que conectan a la lengua con la enunciación, aludiendo a algo o alguien que se muestra (Dasen, Changkakoti, Abbiati, Niraula, Mishra, Foy, 2009).

Si bien una buena parte de los elementos léxicos adquieren sentido pleno a través de su uso contextualizado, en el caso de los deícticos esta situación se ve acentuada al máximo (Calsamiglia, Tusón, 2018); no obstante, existen deícticos que refieren contenidos menos abstractos (por ejemplo, refieren a lugares: aquí, en, allá) y otros que implican contenidos más abstractos y descontextualizados puesto que codifican sistemas de relaciones espaciales (por ejemplo, derecha-izquierda), lo que involucra procesos de representación altamente complejos (Mishra, Dasen, Niraula, 2003).

En términos ontológicos, la deixis espacial es fundamental ya que organiza el lugar en el que se desarrolla el evento comunicativo, señalando los elementos locativos en relación con el espacio que "crea"y así hace emerger al yo como sujeto de la enunciación (Calsamiglia, Tusón, 2018).

Entre las exiguas investigaciones que existen respecto a la articulación teórica y metodológica de sistemas de referencia y deixis, se encuentra el trabajo llevado a cabo por Muñoz y Alonqueo, quienes en 2017 publicaron el artículo "Referencias espaciales lingüísticas y deícticas de niños rurales mapuches y no mapuches: un estudio exploratorio sobre la descripción del trayecto en espacios amplios". En dicha investigación identificaron, a través de su diseño experimental, las referencias espaciales, lingüísticas y deícticas que un grupo de escolares realizó en una única ocasión, para lo cual describieron las expresiones que alumnos rurales mapuches y no mapuches, de cuarto año de enseñanza básica, usaron para referirse a espacios amplios en una tarea de descripción del trayecto escuela-hogar. Participaron 20 niños pertenecientes a comunidades rurales cercanas a la ciudad de Temuco. Se distribuyeron en una submuestra de escolares mapuches y escolares no mapuches. Los niños mapuches tenían como lengua materna el castellano y ninguno de ellos era hablante del mapuzungan. El rango de edad fue entre 9 y 10 años, con un total de 9 niños y 11 niñas. Los resultados mostraron que el espacio fue descrito principalmente a través del sistema sin coordenadas, tanto en niños mapuches como no mapuches, empleando deícticos lingüísticos y gestos referidos al marco de referencia relativo en ambos grupos. El sistema con coordenadas tuvo menor presencia; asimismo, en los escolares mapuches hubo más referencias geocéntricas (usar como referencia espacial los puntos cardinales), a diferencia de los niños no mapuches. Se encontró que la relación de las experiencias cotidianas e información sensorial con la descripción lingüística y gestual del espacio, son factores de variabilidad en la representación y predilección por marcos de referencia espacial entre los hablantes. 
Por su parte, Rimassa y Fernández (2014) llevaron a cabo el trabajo denominado "Conceptualización del espacio y su relación con el desarrollo cognitivo: Un estudio piloto en el español de Chile". Los resultados de la investigación muestran una tendencia creciente en la complejidad de las expresiones lingüísticas; sin embargo, debido a que la investigación contó con una muestra muy pequeña (8 participantes), los hallazgos no se pueden considerar estadísticamente significativos.

\section{El desarrollo cognoscitivo y el espacio}

Para la teoría psicogenética, el desarrollo cognoscitivo responde al modelo de equilibración. La equilibración es un proceso de autorregulación interminable (implicado en la construcción del conocimiento), a través del cual se busca alcanzar la máxima coordinación que puede tener un sistema con el objeto de conocimiento (asimilación/acomodación), la máxima coordinación entre los subsistemas que lo conforman (relaciones parte-parte) y la máxima coordinación entre el sistema como totalidad con los subsistemas (relaciones todo-parte). En otras palabras, el desarrollo cognoscitivo consiste en coordinaciones cada vez más amplias y congruentes de la información en un sistema de conocimiento altamente organizado y equilibrado. En la medida que el sistema se torna más coherente, coordinado y coordinante, organizado y equilibrado, el contenido concreto va cediendo su importancia a contenidos formales, lo que no significa que lo concreto deje de estar presente como fuente de información cuando así se requiera. De esta forma, otra manera de caracterizar el desarrollo cognoscitivo sería como una búsqueda permanente de mayores niveles de abstracción y descontextualización del contenido (Calderón, 2015; Flavell, 1996).

Para Piaget e Inhelder (1947; 2015), el espacio como representación, no puede ser aprehendido como resultado directo y exclusivo de la información sensorial, sino que debe irse elaborando poco a poco a través de la acción simbólica del sujeto, acción que implica la construcción de esquemas de conocimiento cada vez más abstractos. El conocimiento del espacio proviene en principio de la actividad sensoriomotriz, y posteriormente, de un nivel representativo en el que la actividad, real o imaginada, irá flexibilizando, coordinando y haciendo reversible las imágenes -en este caso, espaciales- para convertirlas en operaciones.

En el estadio de las operaciones concretas se desarrolla la reversibilidad del pensamiento. Con ella los niños van siendo capaces de coordinar distintas perspectivas y conocimientos y empezar a entender cómo se afectan unas a otras. En el terreno de la conceptualización espacial se complejizan las relaciones topológicas (cerca-lejos, etc.) y se empiezan a reconocer las propiedades proyectivas de los objetos (predecir qué aspecto tendrá un objeto observado desde diversos puntos de vista o ángulos de visión). Esto le permitirá al sujeto representar objetos tridimensionales en pares de direcciones (derecha-izquierda, arriba-abajo, adelante-detrás), si bien todavía no domina la perspectiva y aún se tienen dificultades para concebir que las rela-

Diólo@os sobre Educación año 12 | número 23 | julio-diciembre 2021 | ISSN 2007-2171 
ciones espaciales de los objetos varían según el punto de vista del observador (como resultado del egocentrismo). Todas estas construcciones, así como la coordinación de variables espaciales entre sí, le permitirán al niño dibujar los objetos en dos dimensiones (a modo de plano), con muchos más detalles que antes y con cierta coordinación, dirección, orientación y escala. Para Piaget e Inhelder (1947), en esta etapa se consolida cierto nivel de descentración espacial, es decir, el niño habrá superado hasta cierto punto el egocentrismo.

En el estadio de las operaciones formales, las operaciones espaciales pueden ser totalmente separadas de la acción física, de modo que ya se es capaz de considerar un universo total de posibilidades y comenzar a pensar sobre nociones como la inconmensurabilidad de la idea de infinito. Se empieza a ser capaz de llevar a cabo representaciones sobre representaciones y de pensar el espacio desde el punto de vista de alguien más. Se reconstruye la noción de espacio euclidiano (sobre la base de las construcciones previas), reconociendo propiedades relacionadas con tamaño y medida del espacio, distancias, direcciones y perspectiva. La capacidad de realizar mapas con elementos coordinados, con escala, con leyes de geometría cartesiana, se adquiere en este periodo.

\section{Método}

Existen pocas investigaciones en torno a la conceptualización del espacio y su expresión lingüística con participantes mexicanos.

El propósito central del presente trabajo es exponer los datos de una investigación en torno a cómo niños y adolescentes entre 9 y 14 años conceptualizaron el espacio (sistema de referencia) y cómo expresaron lingüísticamente (tipo de deícticos se emplearon) tal conceptualización, así como analizar si esta posibilidad de conceptualización está vinculada a la creciente capacidad de coordinar coherentemente la información; en ese sentido, interesaba determinar si existían patrones lingüísticos y cognoscitivos en la forma de resolver la tarea por grupo etario, partiendo del supuesto de que los sistemas de referencia con coordenadas implican un mayor reto cognoscitivo, como se expuso en la sección precedente.

Para dar cuenta de los objetivos perseguidos en la investigación, se retomó el trabajo de Rimassa y Fernández (2014). También se desarrolló un sistema de análisis de las respuestas de los participantes con base en la teoría psicogenética piagetiana (Piaget, Inhelder, 2015), que considera al desarrollo cognoscitivo como una serie de organizaciones y reorganizaciones del conocimiento, siempre en búsqueda de la máxima y mejor coordinación de la información; de esta forma, en este trabajo las respuestas que se consideraron más avanzadas fueron aquellas que lograron coordinar de mejor manera el mayor número de información posible, y de forma cada vez más abstracta y descontextualizada. 


\section{Enfoque y diseño metodológico}

La investigación se realizó bajo un enfoque mixto de temporalidad transversal, con un alcance descriptivo y correlacional en el que las expresiones lingüísticas son consideradas como un indicador de la conceptualización espacial.

\section{Participantes}

Los participantes se encontraban en un rango de edad de 9 a 14 años (tabla 1).

Tabla 1. Edad de los participantes

\begin{tabular}{|c|c|}
\hline Cantidad de niños & Grupo \\
\hline 20 & 9 y 10 años \\
\hline 20 & 11 y 12 años \\
\hline 20 & 13 y 14 años \\
\hline
\end{tabular}

Los criterios de inclusión fueron:

a) Los participantes debían ser monolingües para que no existiera interferencia o efectos de lengua uno (L1) con lengua dos (L2), como se sabe, ocurre en fenómenos y tareas lingüísticas con sujetos bilingües (Martínez, 2005; Li, Macuha, Sousa, Sato, Nanri, 2009).

b) Estar cursando el año escolar correspondiente a su edad: este criterio obedece a la necesidad de controlar tanto edad como escolaridad como índices del nivel de desarroIlo de los participantes. Para Piaget (1973) y Piaget e Inhelder (2015), el periodo de las operaciones concretas instaura un tipo de pensamiento en el que el niño es capaz de una inteligencia reversible, que le permite justamente empezar a trabajar con las operaciones (acciones mentales interiorizadas). En el caso de la tarea propuesta a los participantes era esencial que contaran con esta capacidad, por ello se eligió iniciar a los 9 años y ligar la edad con el grado escolar: un participante de 9 años, en el grado escolar correspondiente, manifestaría sin dudas un pensamiento operatorio concreto. El experimento llegó hasta los 14 años ya que a esa edad los sujetos se encuentran, si están transitando en el grado escolar correspondiente a su edad, en un tipo de inteligencia operatoria formal. De esta forma, este estudio se concentra en la infancia y la adolescencia, controlando edades en las que claramente se trabaja con un tipo de estructura mental particular (estructura operatoria concreta o estructura operatoria formal).

c) Tener un rendimiento escolar promedio: este criterio se adoptó dado que interesaba saber cómo la mayoría de los niños de un grupo escolar afrontaban y resolvían la tarea propuesta, es decir, no interesaba saber cómo lo hacían los sujetos muy hábiles 
o sujetos con cierta dificultad escolar, sino los niños de comportamiento típico (que constituyen la mayoría del grupo). El rendimiento escolar se consideró como variable a controlar, porque se sabe que puede ser uno de los indicadores, desde luego no el único, de la inteligencia (Almeida, Guisande, Primi, Lemos, 2015). El rendimiento escolar de los alumnos se valoró preguntando directamente a los profesores y directivos de la escuela.

d) Estudiar en el sistema de educación pública en la ciudad de Querétaro, México. Interesaba entrevistar únicamente a alumnos del sistema público porque la literatura previa (Calderón, Maldonado, Soto, 2014) muestra que existen diferencias significativas entre el comportamiento y logro escolar de niños de sistemas públicos, en comparación con sistemas privados de educación. En estudios posteriores se podrá replicar la presente investigación con participantes del sistema privado, y posteriormente comparar los datos para valorar qué tanto impacto tiene el sistema educativo al que se acuda sobre la comprensión y comunicabilidad de la ubicación espacial.

La investigación se hizo con perspectiva de género, por lo que la mitad de los participantes fueron niños y la mitad niñas (los resultados relativos al sexo no se exponen en este trabajo). Los grupos constituidos se conformaron siguiendo el criterio de edad y grado escolar.

De acuerdo con el código de ética de la American Educational Research Association (2011), el consentimiento informado por parte de la institución educativa es suficiente cuando la investigación no implica ningún riesgo para los participantes, como sucede en el presente trabajo. Sin embargo, también se contó con la aprobación de los entrevistados, así como la de sus respectivos padres o tutores. Los datos personales de los alumnos (nombre, edad) fueron tratados con absoluta confidencialidad.

Finalmente, es importante señalar que se consideró filmar a los participantes, sin embargo, esto no fue autorizado; con ello se perdió información no verbal y oral suprasegmental (como el tono, ritmo e intensidad de la voz) sobre cómo los participantes conceptualizan el espacio. Información no verbal como los gestos, las posturas corporales, las expresiones faciales, así como las inflexiones de voz, podría aportar datos en torno al nivel de certeza o solidez de los esquemas conceptuales con los que se cuenta para afrontar y resolver una tarea o problema, es decir, podría ser un índice del nivel de evolución y consolidación de un conocimiento en un momento específico del desarrollo ontogenético. Este tipo de información también podría aportar o develar aspectos importantes sobre la cercanía y/o apuntalamiento de los deícticos (tanto de sistemas referenciales sin y con coordenadas) (Muñoz, Alonqueo, 2017) en el cuerpo y los rasgos suprasegmentales de la voz al momento de conceptualizar, interpretar y describir la ubicación de objetos en el espacio físico, tal como ya lo señalaban Sinclair y Gol (2010). Los investigadores enfatizan la relevancia de este tipo de recursos semióticos en la descripción de 
conceptos o interpretaciones que contribuyen a desvanecer la distinción entre la abstracción mental y el mundo físico. Aseguran que el significado y la interpretación en una situación de comunicación -en especial en campos como el matemático, el físico y el espacial- depende en buena medida del movimiento y del tiempo, que a su vez son dimensiones del pensamiento típicamente ausentes en las definiciones formales y escritas. Insisten que el gesto y la palabra (desde lo que se dice hasta cómo se dice) contribuyen de forma diferenciada y única a la conceptualización y comunicabilidad dado su rol de mediadores semióticos.

\section{Diseño de la investigación}

La investigación constó de dos etapas:

a) Etapa diagnóstica: en esta se aplicó la prueba diagnóstica de inclusión, Evaluación Neuropsicológica Infantil 2 (ENI-2), en su sección "habilidades espaciales, comprensión y expresión de derecha e izquierda" (Matute, Rosselli, Ardila, Ostrosky, 2013) para determinar que los participantes contaban con las capacidades espaciales y de lateralidad que requería la investigación, es decir, que se comportaran como lo esperado según su rango de edad.

b) Etapa experimental: a los participantes que cumplieron con lo esperado en la etapa diagnóstica se les propuso la tarea "Descripción de la escena visual" (Rimassa, Fernández, 2014).

\section{Instrumentos, tareas y procedimiento}

Se emplearon dos instrumentos: a) una prueba diagnóstica que consistió en la aplicación de la sección de "Habilidades espaciales, comprensión y expresión de derecha e izquierda" de la ENI-2, y b) la tarea "Descripción de la escena visual". En ambas pruebas se entrevistó de forma individual a los participantes.

El propósito de la prueba del inciso a) (ENI-2) fue determinar si los niños contaban con el nivel de comprensión y expresión de la lateralidad relativo a su rango de edad. Todos los participantes alcanzaron el puntaje esperado de acuerdo con lo que indica ENI-2. Por otro lado, el propósito de $b$ ) "Descripción de la escena visual", fue obtener información sobre la manera en que los participantes conceptualizan el espacio a través de los sistemas de referencia y cómo expresan dicha conceptualización en la lengua.

La tarea del inciso b) "Descripción de la escena visual” consistía en que el participante debía describir la ubicación de una serie de objetos (imágenes de un perro, una mesa, un candelabro, una silla, una muñeca, un refrigerador, un gato) contenidos en una fotografía (escena visual) a una persona que acomodaría dichos objetos tridimensionales (los representados en la fotografía) en una maqueta. El participante suponía que la persona que acomodaba los objetos no

Diálo@os

sobre Educación

TEMAS AC año 12 | número 23 | julio-diciembre 2021 | ISSN 2007-2171 
conocía la fotografía que él describiría y tampoco compartían el campo visual; diversos estudios (Pavez, Coloma, Maggiolo, Martínez, Romero, 2002; Pavez, Coloma, Maggiollo, 2008) muestran que cuando un sujeto debe describir la ubicación espacial de un objeto, se esmerará más si no comparte la información y la escena visual con la persona a quien se la describe.

Participante y acomodador se ubicaban de modo traslacional uno respecto al otro, es decir, ambos mirando en la misma dirección, aunque la persona que acomodaba los objetos se situaba por detrás del alumno, teniendo entre ellos una mampara que impedía ver el espacio del otro. Finalmente, se indicaba al estudiante que ganaría el juego si el acomodo final (ubicación espacial) que se lograra de los objetos en la maqueta quedaba lo más parecido posible al de la fotografía que él había descrito.

La técnica mediante la cual se llevó a cabo el acercamiento empírico para levantar los datos fue el interrogatorio clínico de tipo piagetiano, que implica partir del supuesto de que el que sabe es el participante, y su propósito fundamental es recuperar los datos necesarios para establecer la psicogénesis de las ideas infantiles, a través de una interacción permanente entre las preguntas del investigador y las respuestas de los niños (García, Castorina, 2014).

\section{La construcción del sistema de clasificación}

Con base en los aportes de Levinson $(1996 ; 2003)$ respecto a los sistemas de referencia espacial, se realizó una clasificación general de las respuestas de los niños en dos niveles: sistemas de referencia sin coordenadas y sistemas de referencia con coordenadas (figura 1).

Figura 1. Codificación de los niveles de organización espacial

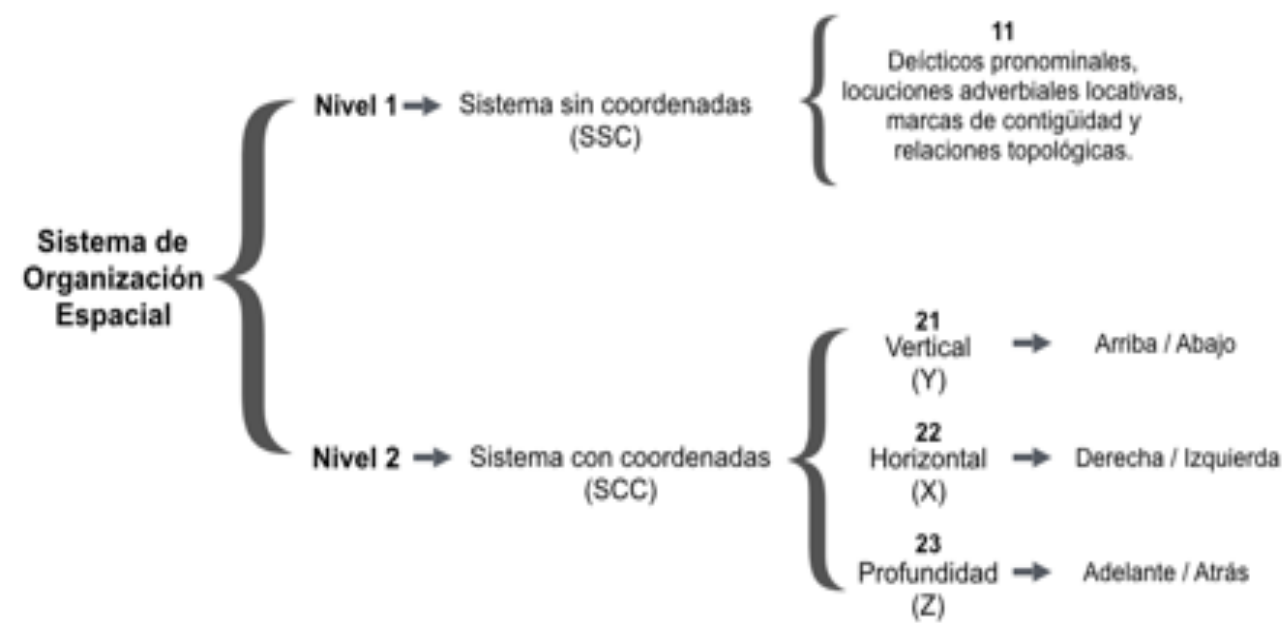


En la clasificación mostrada, los deícticos (Calsamiglia, Tusón, 2018) no vinculados al plano cartesiano quedaron en la codificación de sistema sin coordenadas (número de código 1) y los deícticos vinculados al plano cartesiano quedaron bajo la categoría de sistema con coordenadas (número de código 2); a su vez, cada componente de cada nivel se codificó de la siguiente manera: con el número 11 todos los deícticos pronominales, locuciones adverbiales locativas, marcas de contigüidad y relaciones topológicas dentro del sistema sin coordenadas, y dentro del sistema con coordenadas se codificó con el número 21 al eje vertical (eje "Y") arriba y abajo, con el número 22 al eje horizontal (eje "X") derecha e izquierda y con el número 23 al eje de la profundidad (eje "Z") adelante y atrás.

Se transcribieron los audios de cada participante. Una vez listas las transcripciones, se hizo una segmentación por frases, atendiendo un criterio semántico y pragmático, y se tomó como base la intención del emisor de brindar la ubicación espacial de un objeto determinado (subrayado en cada frase); a continuación, se muestra un ejemplo:

Participante 9

(1) "El refrigerador acá",

(2) "el gatito adelante del refrigerador por donde está la puerta",

(3) "la silla al lado del refrigerador, pero no tan pegada",

(4) "la muñeca en la silla",

(5) "la mesa acá",

(6) "el perrito adelante de la mesa",

(7) "las velas arriba de la mesa, pero en la orilla de acá".

Una vez realizada la segmentación por frases, se construyó la base de datos con el objetivo de registrar lo realizado por los participantes. Dicha base de datos se conformó de 400 frases u oraciones (que constituyen la muestra total de la presente investigación ya que en promedio cada participante produjo entre 5 y 7 frases en la resolución de la tarea). A continuación, se describe un ejemplo.

Tabla 2. Base de datos: Sistema de referencia

\begin{tabular}{ccccc}
\hline Niño & Grupo & Sexo & Frase & Deíctico empleado \\
\hline 43 & 3 & 1 & $\begin{array}{l}\text { eeeehhh la... muñeca está sentada } \\
\text { del lado izquierdo con, de, en la silla, }\end{array}$ & 22 \\
\hline
\end{tabular}

En la tabla anterior se muestran cinco columnas. La primera se refiere a la codificación que se le dio a cada participante (del 1 al 60); la segunda consigna el grupo etario al que pertenece

Diálo@os

sobre Educación año 12 | número 23 | julio-diciembre 2021 | ISSN 2007-2171 
(Grupo 1: niños pequeños; Grupo 2: medianos; Grupo 3: grandes); la columna 3 se refiere al sexo de los participantes: 1 para las niñas y 2 para los niños. La cuarta columna es el texto oral que produjo el niño, y en la quinta columna se consigna la categorización global de la respuesta del niño, en función del tipo de deíctico empleado para dar la ubicación espacial del objeto.

Cabe señalar que cuando los participantes empleaban diversas expresiones lingüísticas para describir la ubicación espacial de un mismo objeto, se consideró el deíctico o expresión más avanzada alcanzada, para asignar el puntaje correspondiente. En el ejemplo anterior, en la columna 5 se clasificó la respuesta del niño como 22, ya que es el deíctico más avanzado (izquierdo) empleado por él en esta descripción.

\section{Resultados}

Los resultados obtenidos se presentan de dos maneras: a) se muestra una primera organización de las respuestas obtenidas por los participantes con base en el contraste "sistema de organización espacial sin coordenadas" y "sistema de organización espacial con coordenadas"; b) se exponen los datos de forma detallada, es decir, el sistema de organización sin coordenadas se subdivide en deícticos pronominales y marcas de contigüidad, y el sistema de organización con coordenadas se subdivide en eje $Y$, eje $X$ y eje $Z$. El propósito de esta segunda manera de organizar los datos fue determinar si los distintos subsistemas de referencia (de un mismo sistema) se comportan entre sí de forma similar o no. Particularmente, interesaba distinguir al plano vertical (eje Y) del horizontal (eje X y Z) ya que la literatura (Levinson, 1996) indica que, si bien el eje $Y$ ubica coordenadas en un plano imaginario (cartesiano) -por lo que se parece a los ejes $X$ y Z-, a nivel de recursos cognitivos involucrados, es decir, del reto que representa su comprensión, este eje se parece más al sistema sin coordenadas; es necesario recordar que los puntos de referencia arriba-abajo del eje Y se mantienen bastante constantes en la interacción con el mundo, no así adelante-detrás y derecha-izquierda (Shepard, Hurwitz, 1984; Tommasi, Laeng, 2012).

Cabe señalar que ninguno de los participantes del estudio empleó un sistema de referencia geocéntrico con coordenadas (aunque esto era posible); sin embargo, no es de extrañar ya que en México las referencias espaciales geocéntricas se emplean únicamente en contextos especializados.

Cuando las respuestas de los participantes son organizadas bajo el contraste más amplio de los tipos de sistemas de referencia, se obtiene la siguiente figura. 
Figura 2. Porcentajes de respuesta de los participantes en sistema de organización espacial sin coordenadas y con coordenadas

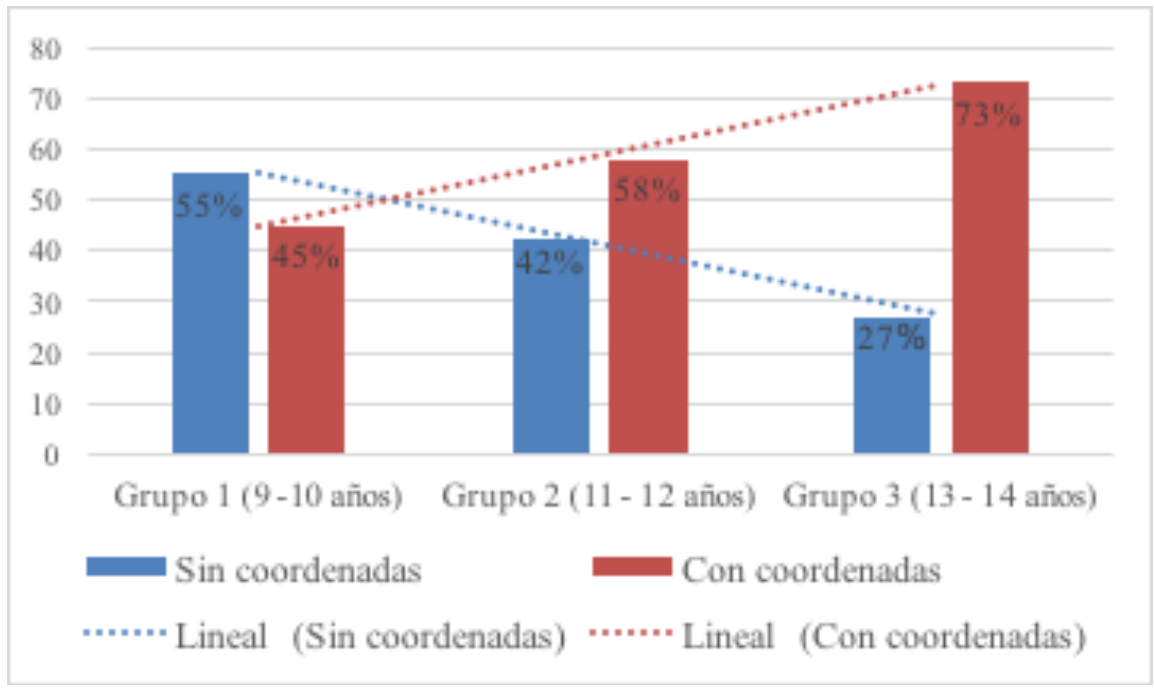

La figura muestra que los participantes más pequeños tienden a emplear el sistema de referencia sin coordenadas, siguiendo este una clara línea evolutiva descendente. A la inversa, los participantes mayores emplean con mayor asiduidad el sistema de organización con coordenadas y también se observa una línea evolutiva, aunque en este caso ascendente (de los participantes más pequeños a los más grandes). Sin embargo, resulta interesante constatar que, si bien los participantes se apoyan más en uno u otro sistema, todos los grupos emplean ambos modos de organización espacial, lo que significa que este tipo de expresiones lingüísticas se mantienen a lo largo de la vida.

Para determinar si las diferencias entre los grupos fueron significativas se empleó la prueba Kruskal-Wallis para poblaciones de distribución no normal, que brinda un análisis global de la varianza de cada grupo para compararla con los otros grupos. El análisis de varianza mostró que efectivamente los grupos se diferenciaban entre sí de forma estadísticamente significativa $(p<0.005)$.

A continuación, se exponen algunos ejemplos para mostrar el modo en que los participantes se apoyaban en los sistemas de organización espacial al generar sus respuestas. Estos proveen un soporte cualitativo al análisis estadístico, ilustrando cómo las expresiones lingüísticas de la conceptualización del espacio evolucionan sistemática y significativamente en relación con el grupo de edad/escolar de pertenencia de los sujetos.

En el primer ejemplo el participante describe, a la persona que acomoda, la ubicación del refrigerador y de la mesa; "El refri acá. La mesa acá" (participante 9, grupo 1). En ambas indicaciones el participante utilizó el deíctico pronominal "acá", que pertenece al sistema de organización sin coordenadas. Como se recordará, al momento de realizar la tarea de "Descripción de 
la escena" no existió contacto visual entre investigador y participante, por lo tanto, la utilización del deíctico pronominal "acá" podría indicar cierta dificultad por parte del niño para descentrarse de su propio punto de vista y considerar el punto de vista del acomodador, ya que este no tenía forma de saber dónde es "acá" sin compartir la información visual con el menor; el egocentrismo de la expresión del niño se origina en gran medida en la dificultad de coordinar la información, su punto de vista y el punto de vista de la otra persona, lo que genera dificultades también para procesar la información de manera más abstracta y descontextualizada.

El siguiente ejemplo corresponde a un niño del grupo 2; “Acomoda el refrigerador del lado izquierdo" (participante 23, grupo 2). En este ejemplo se empleó una expresión locativa relacional: "del lado izquierdo", la cual parte del sistema de organización espacial con coordenadas, dentro del eje horizontal $X$. Sin embargo, izquierda/derecha, al ser deícticos, también requieren explicitación de información en torno a una referencia (a partir de la cual se establecerá la izquierda y la derecha). Dicha referencia no fue establecida por el niño, lo cual denota cierto nivel de egocentrismo y de dificultad para establecer relaciones coordinadas entre el todo y las partes: pareciera que una vez que el participante observó y logró situar una ubicación, ya no le fue posible "salirse" de esa ubicación para considerar otras y así brindar una información más precisa a quien estaba acomodando los objetos.

El último ejemplo pertenece a un adolescente; "El refri primero... en la esquina... a la derecha ..." (participante 48, grupo 3). El participante 48 ubicó el objeto utilizando ambos sistemas de organización espacial: por un lado, las marcas de contigüidad y lugar "en la esquina" (del sistema de organización sin coordenadas) y, por otro lado, "a la derecha" (del sistema de organización espacial con coordenadas en el eje X). Además, agregó una marca ordinal (primero) como para aclarar al oyente que debía iniciar su acomodo en el extremo derecho, en una esquina. En términos psicológicos, coordinar ambos sistemas de referencia permite una descripción más detallada, lo que implica coordinación de distintas informaciones para generar un todo más organizado.

En la figura 3 se exponen los resultados de los sistemas de organización de manera más detallada. En esta figura el sistema de organización con coordenadas se desglosa en X, Y y Z. También se desglosa el sistema de organización sin coordenadas en "deícticos pronominales y locuciones adverbiales locativas" y "marcas de contigüidad y relaciones topológicas". 
Figura 3. Porcentajes de respuestas desglosados de los sistemas de referencia

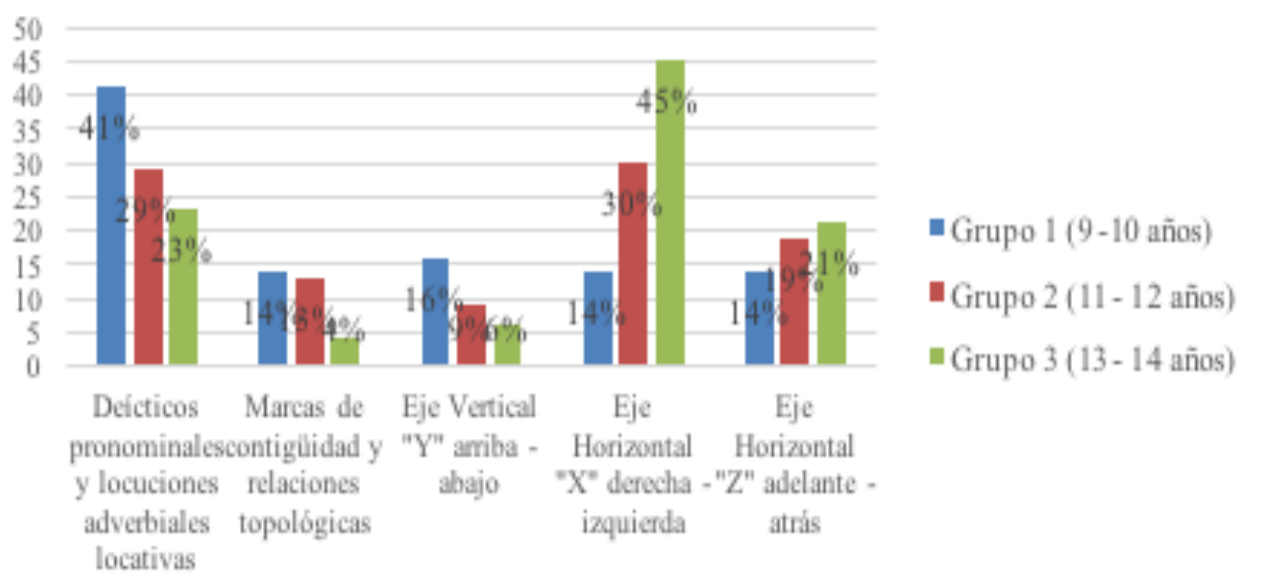

El primer aspecto a destacar de la figura anterior, si se centra la atención sobre las barras relativas al sistema sin coordenadas, es que tanto los deícticos pronominales/locuciones adverbiales locativas y las marcas de contigüidad y relaciones topológicas presentan una curva evolutiva descendente, sin embargo, es evidente que, como estrategia para describir la ubicación espacial, los deícticos pronominales y las locuciones adverbiales locativas son mucho más importantes para los participantes que las marcas de contigüidad y relaciones topológicas.

Si se concentra la atención en las respuestas obtenidas para el eje $Y$, a pesar de ser un eje imaginario vertical, muestran un comportamiento similar (una curva evolutiva descendente) a lo que acontece en el sistema sin coordenadas; es decir, los niños más pequeños tienden a organizar el espacio más frecuentemente bajo este tipo de subsistema que los mayores. Nuestros datos muestran que, efectivamente, y en concordancia con autores como Shepard y Hurwitz (1984) y Tommasi y Laeng (2012), el eje vertical (Y) es más empleado por lo niños pequeños, lo que refuerza la idea de que implica un reto cognitivo menor que el eje horizontal y de profundidad ( $X$ y Z), ya que la ubicación vertical del observador (arriba-abajo) se mantiene constante, es decir, generalmente arriba y abajo no cambian como puntos de referencia, sin embargo, derecha-izquierda o adelante-atrás sí.

Otro contraste que interesa destacar es el comportamiento de las respuestas basadas en los ejes X y Z. Si bien ambos tipos de respuesta presentan una clara evolución ascendente, nuestros datos muestran que la estrategia que involucra al eje $X$ (izquierda-derecha) resulta más importante en la conceptualización y descripción de la ubicación espacial que la que implica al eje Z. Es decir, conforme el desarrollo avanza, lo que implica un pensamiento más coordinado a la vez que mayor experiencia en el mundo, la descripción de la ubicación espacial tiende a descansar sobre el eje horizontal izquierda-derecha, probablemente debido a que, al estar menos relacionada con nuestra experiencia fenomenológica en el mundo, es más distintiva, singular y 
esclarecedora para conceptualizar el espacio y expresar tal conceptualización de forma lingüística.

En cuanto a la diferencia de las variables entre grupos, la prueba Kruskal-Wallis arroja que existe una diferencia global estadísticamente significativa $(p<0.001)$.

\section{Consideraciones finales}

La construcción del conocimiento, del conocimiento espacial y de la propia inteligencia, en el desarrollo ontogenético no es otra cosa que la manera misma en que el ser humano ve, interpreta y organiza el mundo; de forma más precisa: es el largo camino que toma la capacidad de coordinar todas las pistas y elementos de una situación en un todo organizado y coherente. Es plantearse un problema con dispositivos mentales (esquemas) cada vez más sólidos y flexibles que permitan una mayor abstracción y descontextualización de los objetos de conocimiento, una manera de interactuar con el mundo de forma más simbólica y formal (estructura operatoria formal), pero hundiendo sus raíces en el conocimiento sensorial motor (estructura sensoriomotora) (Piaget, 1973).

En este trabajo se expusieron algunos resultados de niños y adolescentes mexicanos a quienes se les aplicó una prueba de descripción de una escena visual (Rimassa, Fernández, 2014) para analizar cómo conceptualizaban e interpretaban el espacio, cómo lograban expresar lingüísticamente este conocimiento por grupos etarios y si era posible identificar patrones 0 esquemas de acción por grupo de desarrollo en la resolución de la tarea.

Los participantes de esta investigación establecieron referencias espaciales (de sistemas con y sin coordenadas) para describir la escena visual, es decir, el espacio fue pensado a partir de la referencia. Sin embargo, la referencia, al menos como se puede establecer en nuestra cultura, es asible e interpretable con base en un sistema de deícticos que permiten organizar el espacio. En la investigación se logró identificar patrones por grupo de desarrollo relativos a la manera en que se piensan y se emplean los sistemas de referencias y los deícticos espaciales vinculados a las posibilidades de integrar y coordinar la información y al nivel de egocentrismo cognoscitivo de los sujetos.

Se recordará que los sistemas sin coordenadas se expresan en elementos lexicales y relaciones deícticas simples y los sistemas con coordenadas lo hacen en elementos lexicales y relaciones deícticas establecidas en el plano cartesiano o en el plano geocéntrico. En el caso del sistema con coordenadas de tipo cartesiano, diversos teóricos (Shepard, Hurwitz, 1984; Tommasi, Laeng, 2012) señalan que primero se conquista el eje imaginario Y o eje vertical (arriba-abajo), seguido del eje $Z$ o de profundidad (adelante-atrás) y finalmente, el que implica una mayor demanda cognitiva es el horizontal, eje X (derecha-izquierda) (Levinson, 1996; Levinson, Wilkins, 2006). Los resultados muestran que, tal como indica la bibliografía al respecto, los participantes del grupo 1 y 2 operan sobre el eje Y, prácticamente igual a como lo hacen con el sis- 
tema sin coordenadas, lo que sugiere que cognitivamente las demandas de estos dos sistemas de referencia son similares.

Aunque lo que se planteará a continuación excede los límites de este trabajo, nos parece altamente relevante señalar que si bien los resultados recién expuestos en torno al eje $Y$ coinciden con la literatura previa que plantea su conquista temprana, también habría que indagar sobre la posibilidad de que, tal como lo consideraba Santos (Panadero, 2000), al ser el espacio una construcción social y encontrarse tan arraigada la metáfora de la verticalidad en nuestro entorno como referente del éxito social y económico, los habitantes de este país (incluidos los participantes de este trabajo), que evidentemente nos asumimos dentro de un espacio social, tendamos a conceptualizar el mundo físico con el esquema social que más familiar nos resulta y con las consecuencias que sobre nuestra persona podría tener el pensar el mundo en términos verticales más que horizontales. Santos plantearía que la verticalidad, a través de la cual entendemos el mundo que habitamos, normaliza relaciones sociales que responden a lógicas de dominación y clasismo, tan arraigadas en nuestro país a través de metáforas como "los de arriba”, "los de abajo", "la clase alta", "la clase baja”, entre otras. Este tema, desde luego abre una veta fecunda de reflexión y debate en la que habrá que pensar el espacio de forma mucho más compleja de lo que se asume cotidianamente, así como las implicaciones que tiene en la manera de relacionarnos como ciudadanos y colectivos humanos, y en la manera de hacer investigación educativa y psicológica.

Respecto al eje $X$ y $Z$, es interesante que el eje $X$ sea la base sobre la que se construyen la mayoría de las respuestas de los sujetos del grupo 3 cuando se centran en el plano horizontal; el eje $Z$ tiene una tendencia similar de evolución al $X$, pero es empleado mucho menos que este. Se podría pensar que el plano $X$ es más importante para los participantes porque les resulta más fácil; sin embargo, coincidimos con la bibliografía sobre el tema y consideramos que $X$ es más demandante cognitivamente; en ese sentido, su mayor frecuencia de aparición podría deberse entonces, a que en términos cognoscitivos es más útil y significativo que el $Z$, es decir, las personas se ven obligadas de forma más frecuente a organizar el espacio en izquierda-derecha que en delante-detrás, por lo que los individuos se ven compelidos a construir de forma más eficaz y rendidora los referentes de $X$ por encima de los de Z. Nuevamente, la noción de espacio abre horizontes para pensar -y quizá replantear- las relaciones individuo-sociedad, cognición personal-cognición cultural-social y sus determinaciones mutuas.

Otro aspecto a destacar es que, dependiendo del nivel de desarrollo de los participantes, es posible observar patrones de conceptualización del espacio más ligados a uno u otro tipo de sistema de referencia y cierto tipo de deícticos; también se observó que, al menos con la población estudiada, nunca se abandonó totalmente ninguno de los sistemas de referencia, es decir, los alumnos mayores continuaron pensando y describiendo el espacio con deícticos como acá, en, Querétaro, etc. En realidad, lo que sucede es un enriquecimiento de las maneras de pensar 
el espacio. Conforme se avanza en el desarrollo, el sistema con coordenadas cobra mayor relevancia; no obstante, nunca se declina del todo el uso del otro sistema, por lo que se puede considerar que la conceptualización y representación del espacio es un proceso continuo a través del cual este se enriquece y complejiza, quizás a lo largo de todo el ciclo vital del ser humano.

Desde la perspectiva teórica (Piaget, Inhelder, 1947; 2015) y de los datos expuestos en el presente trabajo, es posible considerar que lenguaje y cognición se apoyan mutuamente: en sus inicios el espacio como noción se apuntala en el conocimiento sensorial y motor de los sujetos, en la capacidad del niño de encontrar los aspectos invariantes de la situación experimentada; en ese sentido, la cognición precede al lenguaje. Sin embargo, también de manera muy temprana (Vigotsky, 2009), los estímulos lingüísticos empiezan a estructurar el espacio de forma social, primero a nivel interpsicológico, y posteriormente, intrapsicológico o individual. Una vez que lo lingüístico empieza a funcionar de manera intrapsicológica, el lenguaje (lengua oral y escrita) se convierte en el sistema semiótico y representacional más importante para el individuo, confiriéndole a la cognición y a la inteligencia propiedades y capacidades inusitadas. Así, a partir de este momento el lenguaje apoya a la cognición. La inteligencia humana, para ser humana, es lingüística; y el lenguaje humano, para ser humano, tiene que ser inteligente; es decir, no solo es comunicativo, también apuntala al pensamiento y la resolución de problemas, como ha quedado de manifiesto con los hallazgos de la investigación.

Finalmente, en un trabajo como este, en el que se tienen como tópicos esenciales la cognición y el lenguaje, no es posible evitar tener como telón de fondo la pregunta -aún no resuelta- en torno a las relaciones entre estos procesos mentales. Si bien el propósito del presente artículo no es abordar de forma explícita este problema (trinomio basado en la relación lenguaje/ pensamiento-cognición/inteligencia), el planteamiento aquí desarrollado, al poner en tensión la noción de espacio, el desarrollo y su relación con la lengua, puede aportar datos relevantes para alimentar la discusión sobre el trinomio señalado, así como la toma de decisiones didácticas y pedagógicas en la enseñanza y reflexión en torno al espacio y sus consecuencias.

\section{Referencias}

Almeida, L.; M. Guisande; R. Primi; G. Lemos (2015). Contribuciones del factor general y de los factores específicos en la relación entre inteligencia y rendimiento escolar. European Journal of Education and Psychology, 1(3), 5-16.

American Educational Research Association (2011). Code of Ethics. Estados Unidos: Educational Researcher. http://www.aera.net/Portals/38/docs/About AERA/CodeOfEthics\%281\%29. pdf

Calderón, C.; C. Maldonado; M. Soto (2014). Las metáforas y las adivinanzas: Estudio comparativo entre dos entornos escolares. Revista Guillermo de Ockham, 12(2), 51-58. 
Calderón, G. (2015). La metáfora como recurso para comprender la mente infantil. Querétaro: Fontamara/UAQ.

Calderón, G.; L.; Lozada (2020). La conceptualización del espacio: lenguaje y egocentrismo cognoscitivo. Revista Psicumex, 10(1), 75-91. https://doi.org/10.36793/psicumex.v10/1.331

Calsamiglia, H.; A. Tusón (2018). Las cosas del decir: manual de análisis del discurso. México: Ariel. Chatterjee, A. (2001). Language and Space: some Interactions. Trends in Cognitive Sciences, 5(2), 55-61. https//doi.org/10.1016/S1364-6613(00)01598-9

Dasen, P.; N. Changkakoti; M. Abbiati; S. Niraula; R. C. Mishra; H. Foy (2009). Geocentric Gestures as a Research Tool. En Gari, A.; K. Mylonas (eds.). Quod Erat Demonstrandum: From Herodotus'Ethnographic Journeys to Cross-cultural Research. Athens: Pedio Books.

Delval, J. (1995). El desarrollo humano. México: Siglo XXI.

Flavell, J. H. (1996). El desarrollo cognitivo. Madrid: Visor.

Franklin, N.; B. Tversky (1990). Searching Imagined Environments. Journal of Experimental Psychology: General, 119(1), 63-76. https://doi.org/10.1037/0096-3445.119.1.63

García, I.; J. Castorina (2014). Método clínico-crítico y etnografía en investigaciones sobre conocimiento sociales. Cadernos de Pesquisa, 44(154), 1052-1068.

http://dx.doi.org/10.1590/198053142949

Janzen, G.; D. B. Haun; S. C. Levinson (2012). Tracking Down Abstract Linguistic Meaning: Neural Correlates of Spatial Frame of Reference Ambiguities in Language. PLOS ONE, 7(2): e30657. https://doi.org/10.1371/journal.pone.0030657

Levinson, S. C. (1996). Language and Space. Annual Review of Anthropology, 25(1), 353-382. https://doi.org/10.1146/annurev.anthro.25.1.353

Levinson, S. C. (2003). Space in Language and Cognition: Explorations in Cognitive Diversity. Cambridge, Estados Unidos: Cambridge University Press.

Levinson, S. C.; D. Wilkins (2006). Grammars of Space. Explorations in Cognitive Diversity. Cambridge, Inglaterra: Cambridge University Press.

Li, Y.; M. Macuha; E. Sousa; T. Sato; M. Nanri (2009). Cognitive Interference Management in $3 G$ femtocells. 2009 IEEE 20th International Symposium on Personal, Indoor and Mobile Radio Communications. https://doi.org/10.1109/PIMRC.2009.5450030

Lurcat, L. (2014). El niño y el espacio; la función del cuerpo. México: Fondo de Cultura Económica. Majid, A.; M. Bowerman; S. Kita; D. B. Haun; S. C. Levinson (2004). Can Language Restructure Cognition? The Case for Space. Trends in Cognitive Sciences, 8(3), 108-114.

http://dx.doi.org/10.1016/j.tics.2004.01.003

Maravall, C. D. (2007). El espacio y el tiempo en las matemáticas y en la física. Rev. R. Acad. Cienc. Exact. Fís. Nat. (Esp.), 1(2), 259-283).https://rac.es/ficheros/doc/00544.pdf

Martínez, F. (2005). Exploring Figurative Language Processing in Bilinguals: The Metaphor Interference Effect. Doctoral dissertation. Texas: A\&M University. 
Matute, E.; M. Rosselli; A. Ardila; F. Ostrosky (2013). Evaluación neuropsicológica infantil (ENI-2). México: El Manual Moderno.

Mishra, R. C.; P. R. Dasen; S. Niraula (2003). Ecology, Language, and Performance on Spatial Cognitive Tasks. International Journal of Psychology, 38(6), 366-383.

http://dx.doi.org/10.1080/00207590344000187

Moliner, M. (2007). Diccionario de uso del español. Madrid: Gredos.

Muñoz, R.; P. Alonqueo (2017). Referencias espaciales lingüísticas y deícticas de niños rurales mapuches y no mapuches: un estudio exploratorio sobre la descripción del trayecto en espacios amplios. Revista de Lingüística Teórica y Aplicada, 55(1), 73-94.

Panadero, M. (2000). La dimensión temporal en la conformación del espacio geográfico (Leyendo a Milton Santos). Lecturas geográficas. Homenaje a José Estébanez Álvarez. Colección Homenajes de la Universidad Complutense. Madrid: UCM.

Pavez, M.; C. Coloma; M. Maggiolo; L. Martínez; L. Romero (2002). Procedimientos para evaluar discurso (PREDI). Santiago: Ediciones Universidad Católica de Chile.

Pavez, M.; C. Coloma; M. Maggiollo (2008). El desarrollo narrativo en niños. Barcelona: Ars Médica. Pederson, E.; E. Danziger; D. Wilkins; S. Levinson; S. Kita; G. Senft (1998). Semantic Typology and Spatial Conceptualization. Language, 74(3), 557-589. http://dx.doi.org/10.2307/417793

Piaget, J. (1973). Estudios de psicología genética. Buenos Aires: Emecé.

Piaget, J.; B. Inhelder (1947). The Child's Conception of Space. Londres: Routledge y Keegan Paul. Piaget, J.; B. Inhelder (2015). Psicología del niño. Madrid: Morata.

Rimassa, C.; S. Fernández-Silva (2014). Conceptualización del espacio y su relación con el desarrollo cognitivo: un estudio piloto en el español de Chile. Alpha, (38), 137-154.

http://dx.doi.org/10.4067/S0718-22012014000100010

Shepard, R. N.; S. Hurwitz (1984). Upward Direction, Mental Rotation, and Discrimination of Left and Right Turns in Maps. Cognition, 18(1-3), 161-193.

Sinclair, N.; T. Gol (2010). Drawing Space: Mathematician's Kinetic Conceptions of Eigenvectors. Educ Stud Math, 74, 223-240.

Tommasi, L.; B. Laeng (2012). Psychology of Spatial Cognition. Wiley Interdisciplinary Reviews: Cognitive Science, 3(6), 565-580. http://dx.doi.org/10.1002/wcs.1198

Vigotsky, L. (2009). El desarrollo de los procesos psicológicos superiores. Barcelona: Crítica.

Werlen, B. (2017). Action, Knowledge, and Social Relations of Space. En Meusburger P.; B. Werlen; L. Suarsana (eds.). Knowledge and Action. Knowledge and Space, 9, 31-56. Thuringia: Springer Open. 